\title{
LA PHILOSOPHIE DANS LES SCIENCES
}

\author{
Dominique LECOURT
}

La science suscite dans nos sociétés des sentiments dont l'ambivalence n'a cessé de s'accentuer depuis un demi-siècle. Nul aujourd'hui ne défend plus guère l'idée qu'elle pourrait par elle-même résoudre tous les problèmes qui se posent à l'humanité. On en a fini avec la véritable idolâtrie qui avait conduit quelques grands esprits du XIX ${ }^{\mathrm{e}}$ siècle finissant à annoncer qu'elle était appelée à se substituer à la religion pour le plus grand bien de l'humanité.

Il n'empêche que le projet d'une « conception scientifique du monde » reste très vivant. L'idée en particulier qu'il existe une cohérence de l'ensemble de tous les savoirs scientifiques, qui permettrait à terme de parvenir à une maîtrise rationnelle des relations humaines, garde un grand pouvoir de conviction. Les progrès des sciences biologiques depuis cinquante ans, le jaillissement puis l'expansion des biotechnologies, les succès des nouvelles techniques d'information et de communication suscitent l'admiration de nos contemporains.

Mais, dans le même temps, le dénigrement des sciences, qui avait déjà connu un moment fort au début du $\mathrm{XX}^{\mathrm{e}}$ siècle lorsque Oswald Spengler, écrivant Le Déclin de l'Occident, dénonçait la folie de «l'homme faustien », connaît un regain spectaculaire : c'est de peur panique qu'il faut parler face aux progrès mêmes qu'on célèbre par ailleurs comme des prouesses. La menace nucléaire continue de faire l'objet de discours alarmistes. OGM et clonage aidant, le généticien n'est pas loin de prendre figure de malin génie acharné à fausser toutes les valeurs vitales et à falsifier tous les repères éthiques.

La médecine même, naguère régulièrement invoquée lorsqu'il s'agissait de défendre la valeur « progressiste » de la science, devient suspecte. Les industries pharmaceutiques sont réputées la précipiter sur la pente d'une déshumanisation qui fait l'objet d'une déploration rituelle. De l'allongement de la vie humaine - le rêve de Francis Bacon et de René Descartes - on en vient à souligner plus volontiers aujourd'hui les incidences économiques et démographiques néfastes que le surcroît de bonheur qu'il apporterait à qui saurait en bénéficier.

Force est de constater cependant que ce vaste débat social autour de la science ne trouve guère d'écho dans l'enseignement scientifique. Les étudiants peuvent ainsi avoir le sentiment d'un profond hiatus entre la science qu'ils apprennent et la société où ils seront appelés à mettre en œuvre les compétences qu'ils auront acquises au terme d'études extrêmement lourdes.

En tout cas, l'enseignement des sciences, tel qu'il est aujourd'hui conçu, ne leur apporte pas les instruments intellectuels nécessaires pour répondre aux questions qui ne manqueront pas de leur être posées. 
Tout se passe même comme si, par réaction, la pédagogie des sciences dans l'enseignement supérieur s'était raidie. Une image purement calculatoire et opérative de l'activité scientifique tend à s'imposer aux chercheurs eux-mêmes. Ses finalités s'affichent simplement utilitaires. Parce que la science est conçue comme un instrument de puissance et une réserve de certitudes, son enseignement vise essentiellement à la maîtrise technique et récompense souvent non les esprits les plus inventifs mais les plus dociles.

Il y a plus grave encore : les liens qui unissent la recherche scientifique et l'invention technique aux autres formes de la culture humaine semblent avoir été rompus, quand ils ne sont pas résolument niés. Nombreux sont les étudiants qui, dans ces conditions, perçoivent l'enseignement scientifique comme « anticulturel », que ce soit pour s'en réjouir, s'en satisfaire, ou encore qu'ils y trouvent un motif de grave déception, voire de rejet.

La baisse du nombre des inscriptions dans les filières scientifiques des universités, constatée depuis quelques années à l'échelle internationale, trouve sans doute ici une part de son explication. Pour rendre compte de ce phénomène spectaculaire, il ne suffit pas en effet d'invoquer la crainte du chômage ou la modicité des salaires dans les métiers de la recherche, pas plus que l'attrait grandissant des filières de gestion. La ruée, dans notre pays, vers les études de psychologie ou vers les sciences et techniques du sport ne témoigne pas de la part des étudiants d'une rationalité de type purement utilitaire dans le choix des orientations.

C'est bien le contenu et les modalités de l'enseignement scientifique qu'ils mettent en cause, bien en amont de l'enseignement supérieur. Toutes les enquêtes le confirment depuis vingt ans dans notre pays : à mesure que les élèves gravissent les degrés de leur scolarité, leur passion pour les sciences diminue! Les inscriptions universitaires donnent aujourd'hui la mesure de leur désillusion face aux programmes et à la pédagogie qui s'est imposée depuis plusieurs décennies.

Le projet d'implanter ou de développer un enseignement de philosophie des sciences dans les cursus scientifiques répond ainsi à une véritable urgence. S'il y est intégré à part entière et si l'on veille à ce que son contenu soit en prise directe sur les matières scientifiques enseignées, il permettra de remettre en pleine lumière la grande oubliée du scientisme comme de l'antiscience : la pensée scientifique. Si un véritable travail commun s'institue à cette fin entre philosophes et scientifiques, on peut s'attendre à ce que se produise une profonde rénovation de l'enseignement supérieur. Et l'on redécouvrira que cette forme de la pensée communique avec toutes les autres (technique, artistique, politique, éthique...).

Que les ressorts philosophiques de la pensée scientifique soient dégagés et c'est tout une dynamique culturelle qui se trouvera réenclenchée. L'enseignement scientifique retrouvera dans ces conditions son attrait d'aventure intellectuelle aux yeux des jeunes étudiants.

Une rapide enquête sur la situation institutionnelle de l'enseignement de la philosophie des sciences à l'échelle internationale confirme les analyses plus théoriques produites lors du colloque international de $1994^{1}$, Science, philosophie et histoire des sciences en Europe.

1. Science, philosophie et histoire des sciences en Europe, colloque organisé par Dominique LECOURT, les 9 et 10 décembre 1994, à l'École normale supérieure et dans le Grand Amphithéâtre de la Sorbonne, sous la dir. de D. LeCourT, Bruxelles, Commission européenne, 1998 et 2e éd., 1999. 
Dans les pays de tradition anglo-saxonne, la philosophie des sciences présente une tonalité logique accentuée. Sauf rares et brillantes exceptions (Boston University et Cambridge en Angleterre notamment), elle ne fait l'objet de recherche et d'enseignement qu'à l'intérieur de départements de philosophie, et non dans les départements scientifiques. Toutefois, la souplesse des systèmes (avec la pratique des « mineures » qui veut que les étudiants, du moins en début de cursus, s'inscrivent dans plusieurs disciplines) favorise l'acquisition d'une compétence en épistémologie. Mais, c'est surtout le mouvement inverse qui est encouragé : les philosophes des sciences sont incités à acquérir une réelle compétence scientifique ; un grand nombre d'entre eux ont d'ailleurs une formation initiale scientifique et se sont orientés vers la philosophie au niveau du master.

Dans l'Europe continentale, on trouve en Allemagne, qui elle aussi pratique le système des «mineures », quelques postes de philosophie des sciences implantés dans des départements ou facultés scientifiques. Et il existe également dans ce pays un important réseau d'instituts d'histoire de la médecine qui prend en charge les questions de philosophie de la médecine et d'éthique médicale.

C'est sans doute en Italie que se sont exprimées récemment des préoccupations qui rejoignent les nôtres, même s'il n'existe guère à l'heure actuelle d'enseignement d'épistémologie dans les facultés des sciences et de médecine. Le rapport d'un groupe de travail ministériel publié en mars 1998 fait valoir, arguments historiques à l'appui, qu'un enseignement de philosophie des sciences s'avérerait bénéfique pour l'enseignement scientifique à tous niveaux. Mais il n'a pas, à notre connaissance, été, pour l'instant, suivi d'effets significatifs.

Peut-être la France se trouve-t-elle en définitive paradoxalement bien placée du fait de l'histoire propre de son enseignement philosophique pour prendre la tête d'un mouvement de grande portée institutionnelle autant qu'intellectuelle. Le premier effet d'un tel mouvement serait de restaurer l'idée même d'Université moderne qui n'a jamais pu s'implanter dans notre pays et qui subit une crise profonde depuis plus de trente ans à l'échelle internationale.

L'Europe continentale a en effet inventé, à l'extrême fin du XVIII ${ }^{\mathrm{e}}$ siècle, un concept moderne d'université. Un philosophe parmi les plus puissants, Emmanuel Kant, en a tracé le premier dessin en 1798. Un savant parmi les plus universels, Wilhelm von Humboldt, en a réalisé concrètement le prototype à Berlin en 1810, tirant les leçons de discussions fiévreuses où s'illustrèrent, parmi d'autres, les grands noms de Fichte, Schelling et Hegel. Cette Université tournait le dos aussi bien au très ancien modèle anglais qu'au modèle napoléonien.

Dans la pensée de ses inventeurs, deux caractéristiques la distinguaient fondamentalement de toute autre. La première tenait à ce que l'Université devait être, selon le mot de Hegel, « encyclopédie en marche » : lieu, par excellence, du développement des connaissances, miroir vivant de leur agencement dans le savoir contemporain. Elle devait inciter les étudiants à participer le plus tôt possible à cette marche tout en leur apprenant à s'orienter dans la pensée.

La seconde caractéristique tenait à ce qu'elle devait comprendre en son sein une instance philosophique. Cette instance se voyait attribuer la mission de constituer un lieu de réflexion collective où la communauté universitaire trouverait le loisir d'examiner librement les perspectives intellectuelles, mais aussi économiques, politiques et éthiques des progrès du savoir. 
Grâce à une telle instance philosophique, l’Université était censée pouvoir ajuster son point de vue sur elle-même en situant son travail dans l'histoire dont elle était l'héritière. Cette instance devait également lui permettre de procéder au réglage de ses rapports avec l'extérieur : avec l'État, comme le rappelait Kant en délicatesse avec Frédéric II, mais aussi avec le monde économique qui commençait à demander que la recherche et l'enseignement fussent finalisés en fonction de besoins qui se révélaient souvent contradictoires entre eux.

Il se pourrait que le malaise chronique, qui affecte depuis plus de trente ans les institutions d'enseignement supérieur dans l'Europe entière, tienne secrètement à ce qu'ait été depuis longtemps perdu de vue ce concept d'Université, lequel constitue pourtant le soubassement intellectuel de l'existence des institutions qui s'en partagent le titre.

La situation de l'enseignement supérieur en France présente des caractères très particuliers. Le système des Grandes Écoles avec leurs réseaux de classes préparatoires a hypothéqué la fonction sociale des universités, le développement des grands organismes de recherche a pesé sur leurs missions intellectuelles. La création des nombreuses institutions qui - du Collège de France à l'École des hautes études en sciences sociales - se sont, au fil des siècles, établies sur leurs marges a sanctionné leurs carences et a contribué à les aggraver.

Quant à l'instance philosophique, elle n'y a jamais joué le rôle qui aurait dû lui revenir. L'existence d'un enseignement philosophique dans les classes terminales des lycées a eu pour effet de vouer l'essentiel de l'activité des départements de philosophie à la préparation aux concours de recrutement. Le contenu de l'enseignement étant pour l'essentiel tributaire des programmes de l'agrégation, la part de l'histoire de la philosophie n'a cessé d'y croître. Les relations étroites qui auraient dû s'instituer, avec les mathématiciens, les physiciens et les biologistes n'ont jamais connu l'intensité intellectuelle qui aurait pu dynamiser l'Université tout entière.

Si l'on créait aujourd'hui les conditions pour que s'enclenche une telle dynamique, on voit très bien que, du fait de la position de l'enseignement philosophique dans les lycées, c'est l'ensemble du système éducatif qui en tirerait bénéfice, pour peu qu'on prenne les mesures appropriées en matière de formation des enseignants. On pourrait même voir les philosophes apporter leur indispensable contribution à l'élucidation des présupposés philosophiques à l'œuvre dans la pensée des spécialistes en sciences humaines et sociales. Au lieu de tenir ces disciplines pour de perverses rivales et de cultiver à leur endroit une mentalité obsidionale, comme c'est trop souvent le cas dans notre pays, la philosophie jouerait alors pleinement son rôle critique et constructif.

La pensée des citoyens vis-à-vis de la science, devenue l'un des plus puissants organisateurs de la société, gagnerait à cette dynamique quelque allure de liberté.

\section{Dominique LECOURT* \\ (novembre 2000).}

\footnotetext{
* Dominique Lecourt est président du Comité d'éthique de l'Institut de recherche pour le développement (IRD) et professeur de philosophie à l'université de Paris 7 où il dirige le centre GeorgesCanguilhem (case courrier 7041, 2 place Jussieu, F-75251 Paris Cedex 05).
} 